\title{
Silicosis and lung cancer
}

Keywords: silicosis, lung cancer, IARC, crystalline silica, smoking

\section{Introduction}

Silica is a group of minerals composed of silicon and oxygen, the two most abundant elements in the earth's crust. Silica occurs most commonly in a crystalline form, and more rarely an amorphous form. The three main crystalline forms are quartz, cristobalite, and tridymite. Quartz is so abundant and is frequently used in term of crystalline silica. Silicosis is caused by exposure to respirable crystalline silica dust, a basic component of sand, soil, granite, stone, concrete, brick, and most other types of rock that is used in numerous industries, including mining, construction, and manufacturing. Crystalline silica is used as an abrasive blasting agent. Silicosis can cause progressive, disabling and frequent fatal lung disease. Cigarette smoking adds to the lung damage caused by silica. There are more than 100 studies conducted to date have demonstrated that there is "strong and consistent evidence that silica exposure increases lung cancer risk".

The 1975 NIOSH criteria document recommended a reduction in the occupational exposure standard for crystalline silica to $50 \mu \mathrm{g} /$ $\mathrm{m}^{3}$ without mention of cancer in man or animals whereas the World Health Organization (WHO) report of 1986 proposed a health-based limit of $40 \mu \mathrm{g} / \mathrm{m}^{3}$ without reference to carcinogenicity. Evidence for the carcinogenicity of crystalline silica to humans is indeed limited, although credible, alternative explanations, such as bias, chance, or confounding factors have not been adequately excluded. The International Agency for Research on Cancer's (IARC) assesses silica as a probable human carcinogen based on the three evidences: 1) positive experimental rat model of pulmonary carcinogenesis via inhalation and injection, 2) elevated cancer (mostly lung) among silica-exposed workers, and 3) elevated pulmonary cancer risk among workers with extensive silicosis, frequently diagnosed and recorded in conjunction with compensation registers (IARC, 1987). IARC demonstrated that the following epidemiology issues were of concern to the scientific communities on the Monograph Writing Committee several years ago: 1) absence of dose-response findings, 2) lack of adjustment for smoking, lack of adjustment for other possible confounding factors (i.e. arsenic, polycyclic aromatic hydrocarbons), and 4) diagnostic bias of lung cancer among compensated silicotics. For these reasons, the evidence of lung cancer among humans was judged to be limited.

A previous study in 2,209 white South Africans between 1968 and 1986 indicated a positive association between silicosis of hilar lymph nodes and lung cancer. Workers compensated for silicosis outside the mining industry are at an increased risk of developing lung cancer. According to the United States Occupational Safety and Health Administration (US OSHA), around 2.2 million US workers are exposed to silica particles each year. Silica can also cause other health issues for the million of workers who are or have been exposed to it, most notably silicosis, a disabling lung disease. In August 2013, the US OSHA proposed a new rule that would cut around $50 \%$ of the amount of inhalable crystalline silica particles a worker can be exposed to in 8-hour shift. The US OSHA estimates that doing so could prevent 1,600 new cases of silicosis and save nearly 700 lives per
Volume 4 Issue 6 - 2017

\author{
Cheepsattayakorn A, ${ }^{1,2}$ Cheepsattayakorn $\mathrm{R}^{3}$ \\ ' $10^{\text {th }}$ Zonal Tuberculosis and Chest Disease Center, Thailand \\ ${ }^{2}$ Department of Disease Control, Ministry of Public Health, \\ Thailand \\ ${ }^{3}$ Department of Pathology, Chiang Mai University, Thailand
}

Correspondence: Attapon Cheepsattayakorn, I0th Zonal Tuberculosis and Chest Disease Center, I 43 Sridornchai Road, Changklan Muang, Chiang Mai, 50100 Thailand, Tel 6653 I40767/66 53 276364, Fax 6653 140773/66 53 273590,

Email Attapon1958@gmail.com, attaponche@yahoo.com

Received: November 26, 2017 | Published: November 30, 2017

year. Health providers play an active role in protecting workers from silica illnesses. Clinicians should ask patients about their work history to determine if silica exposure has occurred, and if it has, they should implement the early detection of silicosis and lung cancer. Physicians should also encourage patients exposed to silica who also smoke to quit, as smoking and silica exposure together create a greater risk for lung cancer than either factor alone. Furthermore, silica-exposure data are necessary if a threshold value is to be assessed in order to reduce the silicosis risk and lung cancer risk. In addition to risk for lung cancer, risk for tuberculosis, chronic obstructive pulmonary disease (COPD), bronchitis, scleroderma, and possible renal disease are also included.

In conclusion, now, there are several large bodies of evidence to demonstrate and conclude that occupational silica-dust exposure is carcinogenic to humans. Further research is needed for understanding the complex pattern of interactions contributing to lung cancer among silica-exposed workers and to understand whether and to what extent other workplace lung carcinogens, total respirable dust and total surface size and age of silica particles affect the carcinogenic potential of silica. Additionally, the apparent paradox of a lower lung cancer risk in some workplaces with high level silica exposure needs further investigation. Novel epidemiological approaches with incorporation of more detailed exposure information are required. The importance of preventing silicosis and encouraging smoking cessation in reducing lung cancer incidence among silica-exposed workers is highlighted. In order to reduce the risk for silicosis and lung cancer, the silica exposure has to be lowered, with a peak silica exposure below $0.15 \mathrm{mg} / \mathrm{m}^{3}$. By 2025 , silica exposures will contribute very much less than $1 \%$ of all future cancers.

\section{Acknowledgements}

None.

\section{Conflicts of interest}

None.

\section{Funding}

None. 